\title{
EL MEDIO AMBIENTE ANTE LA RACIONALIDAD INDUSTRIAL
}

\section{ENVIRONMENT BEFORE INDUSTRIAL RATIONALITY}

\author{
Wilmer Casasola \\ Instituto Tecnológico de Costa Rica
}

Recibido: 6 de junio de 2013 • Aceptado: 11 de setiembre, 2013

\section{Resumen}

Este artículo discute las implicaciones de la racionalidad industrial en la cultura actual. Contextualiza el análisis con el problema ecológico de los desechos nucleares, en los que el medio ambiente funge como basurero nuclear. Toma como referente teórico para el análisis ético la noción de heurística del temor, de Hans Jonas

Palabras clave: Racionalidad, racionalidad industrial, tecnociencia, residuos radiactivos, heurística del temor.

\section{Abstract}

The paper discusses some problems related to industrial rationality in the current culture. The ecology problem is contextualized along with nuclear waste and the environment as a nuclear dump. As theoretical frame for an ethical analysis this paper takes the Hans Jonas` heuristic notion of fear.

Keywords: Rationality, industrial rationality, tecnosciencie, radioactive waste, heuristic of the fear 


\section{La razón industrializada}

oda racionalidad se caracteriza por su capacidad de fundamentar
un discurso. Hay todo un componente metódico en la elaboración
discursiva. El discurso constituye una pieza de oratoria, con lo que debe ser elocuente y emotivo, con la finalidad de conmover al público que los escucha. También debe contener una fuerza o estructura lógica que permita la coherencia al momento de expresar las ideas. Esta fuerza o estructura lógica predicativa tiene por finalidad enunciar y demostrar la verdad en un argumento, con lo que la tarea de la demostración consiste en exponer las razones de una afirmación determinada. El discurso es un procedimiento racional que organiza al pensamiento con una finalidad práctica. El discurso es el producto de una lógica, de una racionalidad cultivada.

En el contexto en el que aquí se van a emplear los términos racionalidad tecnológica, racionalidad instrumental, racionalidad económica, remiten, más que a la capacidad de fundamentar, a la capacidad de actuar sobre la realidad para transformarla significativamente. Por supuesto, que cualquiera de estas racionalidades tiene su fundamento principalmente en categorías como eficiencia, productividad, utilidad, riqueza, entre algunas otras no consideradas aquí. La racionalidad, en este sentido, remite a la capacidad de incrementar las utilidades de forma eficiente al menor coste posible.

Se podría hablar, a la par de estas racionalidades señaladas, de una racionalidad industrial. La racionalidad industrial es la actividad mercantil que desarrollan las grandes corporaciones multinacionales con miras a cuantificar la riqueza de los accionistas que las sustentan. En esto no hay mayor diferencia con la lógica que opera en la visión capitalista.

La racionalidad industrial repercute negativamente en el medio ambiente cuando en sus políticas de acción está ausente un compromiso ético; o lo que suele llamarse en administración de negocios, responsabilidad social. Uno de los problemas fundamentales de contaminación ambiental deriva, precisamente, de la industria, o más puntualmente, de esta racionalidad industrial. La industria como tal, obedece a políticas económicas capitalistas que en algún grado se apartan del criterio ético en sus acciones.

La finalidad de adquisición de mayores utilidades está por encima de cualquier criterio ético en la racionalidad que mueve a la industria. 
La racionalidad aquí presente es instrumental. La expresión racionalidad instrumental puede definirse de varias formas, todas ellas, sin embargo, apuntan a un mismo tema en común: un cálculo racional cuyo marco de acción es la eficiencia y eficacia orientado hacia la obtención de un fin bajo cualquier criterio. De acuerdo con lo anterior, la racionalidad instrumental establece que "para fines dados deben aplicarse los medios más eficientes posibles" (Burgos, 2003, p. 97). Esto es lo que suele llamarse la teoría medios-fines de Weber.

Para esta teoría instrumental medios-fines, a juicio de Rodríguez (2002), "lo racional es lo eficiente". En este sentido, el punto fundamental lo constituye la racionalidad medio-fin.

Las decisiones más racionales serán aquellas que produzcan mayores beneficios económicos, pues ellas logran el propósito buscado. En consecuencia, esas decisiones son las más eficientes (Rodríguez, 2002, p. 127).

También esta teoría instrumental, o razón instrumental, se hace acompañar de una racionalidad económica, en la que precisamente la determinación costo-beneficio se hace más evidente.

Tanto la racionalidad tecnológica como la económica, convergen en esta relación medios-fines de la teoría instrumental, al considerar que los gastos mínimos y la consecuente maximización del rendimiento en la búsqueda de un efecto deseado, son los factores determinantes de lo lógica industrial. Se cree que en la racionalidad tecnológica, tanto la eficiencia como la fiabilidad son componentes esenciales, puesto que en el criterio económico, variantes como costes, beneficios y función de utilidad son igualmente relevantes. El punto aquí es que tanto la una como la otra, son en esencia racionalidades de orden instrumental al servicio de la industria, y por ello mismo, contenidas dentro de una racionalidad industrial.

Se puede decir, en consecuencia, que la racionalidad industrial contiene a ambas racionalidades. Esto es así porque en el mundo de la industria están presentes factores tanto económicos como tecnológicos; y en cuanto a tecnologías, importan aquellas que maximicen enteramente la marcha industrial y en consecuencia sus utilidades: 
"De este modo, las tecnologías útiles serán aquéllas susceptibles de crear innovaciones que incrementen la eficiencia, la productividad $y, \ldots$ las tasas de ganancias [de una determinada industria]". (Rodríguez, 2002, p. 97).

Estas afirmaciones conllevan a deslegitimar y negar por completo la vieja tesis de la supuesta neutralidad de la ciencia. La tecnología tampoco es neutral, pese a los esfuerzos que muchos hacen en depositar la responsabilidad total en manos del usuario tecnológico, la tecnología busca "alcanzar un resultado predeterminado intencionalmente"; esto es, hay la intención de "obtener un producto, transformar un medio, dominar ciertos eventos." (Coronado, 2002, p. 25). Como señala Rodríguez (2002, p. 101):

La tecnología occidental es considerada como "neutra"; por tanto, de validez y aplicación universales. Se trata de una abstracción impropia que desconoce los contextos sociales, culturales y ambientales específicos....

El engarzamiento entre ciencia y tecnología da lugar a la llamada tecnociencia cuyo foco de acción consiste en la transformación de la realidad a través de múltiples manifestaciones. La tecnociencia es el marco de acción propio de la racionalidad industrial.

El tema de la racionalidad industrial está, pues, íntimamente ligada con la maximización de las utilidades o de la productividad. La instrumentalidad de la ciencia y la tecnología, bajo el marco de acción de la racionalidad industrial, coloca el interés fundamental en la maximización de la riqueza sobre cualquier otro interés social. De acuerdo con André Gorz (El nuevo orden del día), se dice, en el contexto de la razón instrumental, que:

Las acciones son racionales económicamente en tanto que apuntan a la maximación de la productividad. Pero esto solo es posible bajo dos condiciones: 1) la productividad debe separarse de la singularidad individual del trabajador y debe ser calculada a modo de cantidad calculable y mensurable; 2) el objetivo económico de la maximación de la productividad no puede subordinarse a ningún objetivo social, cultural o religioso sino económico, tiene que ser posible perseguirlo sin piedad. (Rodríguez, 2002, p. 127). 
Cuando los intereses sociales, culturales y ecológicos están subordinados a intereses estrictamente económicos, estamos en presencia de una racionalidad industrializada, de una cultura industrializada. La razón instrumentalizada es la razón por excelencia de la proyección capitalista. Una visión radical del capitalismo pretendería subordinar los intereses sociales a los intereses instrumentalmente económicos. Pero, no como una economía política estatal, sino precisamente, como una economía al margen de la intervención estatal. Lo que está en juego aquí es una serie de valores del capitalismo que determinan ciertas acciones a nivel social. Como señala Francisco Rodríguez:

Tanto el capitalismo como el socialismo histórico han estado dominados por ciertos valores, entre los que se encuentran la racionalidad, la eficiencia, la objetividad, las nociones de progreso y crecimiento y las funciones asignadas a la ciencia y la tecnología como medios idóneos de optimizar la explotación de los recursos naturales y de aumentar la productividad. (2002, p. 118)

Razón instrumental y economía política liberal son categorías englobadas en la ideología capitalista, que se mueve bajo la premisa de una racionalidad industrial que antepone la utilidad como valor de las acciones morales. De ahí que, como sostiene Rodríguez (2002, p. 128), "el capitalismo se orienta por la maximización de las ganancias y todo ha de ser subordinado a esa ley fundamental..."; y aquí hay que tener presente que la tecnología juega un papel preponderante en la consecución de esta racionalidad instrumental capitalista. Puntualmente, en el caso de la tecnología, en el contexto de esta razón instrumental, según Rodríguez:

Es utilizada como el medio ideal para incrementar la eficacia y la racionalidad en los procesos productivos, con la consecuente intensificación en la explotación de los recursos naturales. Los aumentos en la productividad se realizan a costa del impacto desequilibrante sobre los ecosistemas. De este modo, la ciencia y sus aplicaciones tecnológicas se transformaron en un elemento esencial para las doctrinas del crecimiento (2002, p. 103).

¿Cuáles son las implicaciones de esta razón industrializada? Básicamente, la instrumentalización de todo, en cuanto genere utilidades. La denominada naturaleza es, sin duda alguna, la fuente por excelencia de 
instrumentalización. Materializando aún más la noción de naturaleza se podría hablar de la tierra como principio fundamental de donde se extrae todo tipo de utilidad. Ahora bien, la paradoja que encierra la razón industrializada con su pretensión de hacer posible la vida humana, es que niega el principio mismo que la sustenta, a saber, la tierra, de donde extrae sus ganancias: a la vez que pretende abastecer a la humanidad de sus necesidades prioritarias (muchas de ellas inventadas y condicionadas), le quita a la vez la fuente de su existencia, la tierra misma, su ecosistema, con lo que se hace evidente la directa responsabilidad de la industria en el deterioro de este recurso vital.

En este contexto hay que señalar la responsabilidad directa de los agentes políticos en el deterioro de los ecosistemas, al ser ellos quienes aprueban derechos y concesiones a las grandes industrias transnacionales para su irracional explotación. El impacto que estas ocasionan, luego de esquilmar el factor tierra, son daños irreversibles en la mayoría de los casos. De ahí se sigue que el crecimiento de la economía y el medio ambiente están en conflicto. Ante un escenario como este, por motivo de los intereses de la economía política liberal, la racionalidad industrial, el ambiente enfrenta una encrucijada incierta que la orienta hacia un cadalso nefasto. A pesar de ello, se abriga la esperanza de que algunos sectores económicos sean compatibles con el medio.

Como explotación del factor tierra, o en general, de los recursos naturales, Leono Bifani (Medio ambiente y desarrollo) sostiene que "Cada tecnología forma parte de un modo de producción específico, que explota el sistema natural y establece un vínculo de interdependencia entre el sistema socioeconómico y su sistema natural." (Rodríguez, 2002, p.102). Desdichadamente, esta interdependencia no es dialéctica, sino que existe o se da una subordinación explotativa de uno hacia el otro. Hay que tener presente que la categoría "socioeconómico" no remite necesariamente a una buena conciencia económica social. Perfectamente, el espacio socioeconómico puede enfocar esta razón instrumental. La relación dialéctica industria-medio ambiente requiere de toda una resemantización de la noción razón instrumental. Sobre todo cuando existe riesgo de daño ambiental. En este sentido, como señala Bifani: 
Desde el punto de vista ambiental, hay que tener presente que cada proceso tecnológico se desarrolla con vistas a objetivos concretos, entre ellos los de explotación de un determinado sistema natural y sus recursos (Rodríguez, 2002, p. 102).

\section{Medio ambiente: un basurero nuclear}

Si bien es cierto que, como han señalado muchos, la energía nuclear ofrece múltiples ventajas, tales como ser competitiva y garantizar el abastecimiento en energía evitando que se acaben las reservas de combustibles fósiles y limitando las emisiones de gas carbónico, también es cierto que la gestión de los residuos de alta actividad son uno de los problemas más graves que presenta la producción de la energía nuclear, y que a la fecha ningún país del mundo ha logrado resolver de forma satisfactoria. Se considera, en efecto, que el almacenaje de desechos radiactivos es una de las cuestiones que más enfrentamientos provoca entre los ecologistas, algunos sectores sociales, las industrias nucleares y sus consumidores, precisamente por el peligro de fugas radiactivas.

Se entiende por desecho radiactivo, de acuerdo con la Agencia Internacional de Energía Atómica, "toda materia que contiene radionúclidos en una concentración superior a los valores que las autoridades competentes consideran admisibles". Entiéndase por radionúclido al "isótopo de un elemento químico que posee la propiedad de emitir radiactividad" (Badillo y Pérez, 2004, p. 63-64)

Un punto crucial en el manejo de la energía nuclear consiste en su tratamiento. Una solución posible es el Almacenamiento Geológico Profundo (AGP), que consiste en enterrar a 500 metros bajo tierra y para siempre, los residuos de las centrales nucleares, los cuales están activos por miles de años. También se habla del Almacén Temporal Centralizado (ATC), que tiene una vida útil de unos cien años, en el manejo de estos desechos. Sin embargo, se estima que el ATC no es suficiente para el tratamiento de desechos radiactivos, si se toma en cuenta la singular característica de los residuos de alta actividad radiactiva de las centrales nucleares, que pueden durar miles de años contaminando de forma irreversible el medio ambiente. De ahí que se apueste mejor al AGP, dado que se lograría aislar bajo tierra grandes cantidades de residuos nucleares el tiempo necesario para que se elimine su radiactividad, evitando 
con ello que se contamine el medio ambiente. No obstante, esta tecnología no garantiza el tratamiento óptimo de los desechos nucleares, si se toma en cuenta que la Tierra es un planeta cambiante, es decir, sus capas geológicas sufren cambios significativos, con lo que no sabríamos, con certeza, lo que podría suceder a largo plazo ,en el subsuelo de la misma.

Diferentes medios estiman la cantidad de desechos nucleares en el mundo. Así, se estima que "cada año se generan en el mundo unas 10.500 toneladas de residuos radiactivos, suficientes para llenar un campo de fútbol con una capa de un metro y medio" ("Residuos nucleares en almacenes...", s.f.). La cantidad de desechos estaría dividida en diferentes actividades. Se considera que

Las centrales de energía nuclear son las que mayor cantidad de estos productos emplean, pero también muchas aplicaciones de la medicina, la industria, la investigación, etc. emplean isótopos radiactivos y, en algunos países, las armas nucleares son una de las principales fuentes de residuos de este tipo ("Residuos radioactivos", s.f.).

Los residuos radiactivos cumplen con dos características fundamentales: peligrosidad y duración. Aunque se trate de pequeñas cantidades, el impacto que ellas tienen sobre la salud humana y no humana está lo suficientemente demostrado. Por otro lado, sabemos que estos isótopos emitirán fuertes radiaciones durante miles de años, con lo que la preocupación por su alto grado contaminativo es aun más alarmante:

Los residuos nucleares o radiactivos son material de desecho generado en el ciclo nuclear, que comienza con la propia extracción del mineral (uranio) utilizado en las centrales nucleares. ("Residuos nucleares: ¿qué hacer con ellos?, s.f.)

Dado que se habla de estas dos características de los residuos radiactivos, a saber, su duración y peligrosidad, se hace necesario, en consecuencia, tener claro el grado de contaminación que presentan estos radiactivos a fin de conocer con claridad qué se entiende por radiación nuclear. En este sentido, se puede hablar de dos grandes grupos de estos residuos radiactivos. ${ }^{1}$

1 Los puntos a) y b) son tomados de "Residuos radiactivos", s.f. 
a) Residuos de alta actividad. Son los que emiten altas dosis de radiación. Están formados, fundamentalmente, por los restos que quedan de las varillas del uranio que se usa como combustible en las centrales nucleares y otras sustancias que están en el reactor y por residuos de la fabricación de armas atómicas. También algunas sustancias que quedan en el proceso minero de purificación del uranio son incluidas en este grupo. En las varillas de combustible gastado de los reactores se encuentran sustancias como el plutonio 239 (vida media de 24400 años), el neptuno 237 (vida media de 2130000 años) y el plutonio 240 (vida media de 6600 años). Se entiende que el almacenamiento de este tipo de residuos debe ser garantizado por decenas de miles de años hasta que la radiactividad baje lo suficiente para que dejen de ser peligrosos.

b) Residuos de media o baja actividad. Son los que emiten cantidades pequeñas de radiación. Están formados por herramientas, ropas, piezas de repuesto, lodos, entre otros de las centrales nucleares y de la universidad, hospitales, organismos de investigación, industrias y varios más.

El desmantelamiento de las centrales nucleares produce grandes cantidades de residuos radiactivos de los dos tipos. Las centrales envejecen en 30 o 40 años y deben ser desmontadas. Los materiales de la zona del reactor son residuos de alta actividad en gran parte y otros muchos son de media o baja actividad.

La gestión de los residuos radiactivos depende de su grado de contaminación. Así, los residuos de baja actividad, diluidos lo suficientemente, son echados a las aguas en pequeñas concentraciones para evitar que dañen al ambiente.

Los índices de radiación que dan estos vertidos son menores en comparación con los que suelen dar muchas sustancias naturales o algunos objetos de uso cotidiano como la televisión. Por el contrario, los residuos de media o baja actividad son almacenados en contenedores durante cierto tiempo hasta el momento en que pueden ser tratados en vertederos de mayor seguridad. Se estima que hasta inicios de la década de los noventa, la industria que manipula materiales radiactivos vertía en barriles los desechos y los arrojaba al mar. 
La procedencia de los desechos radiactivos es diversa. Se trata principalmente de todas las aplicaciones energéticas en las centrales nucleares, especialmente en aquellas plantas encargadas de generar energía eléctrica. El combustible que queda como residuo es lo que genera la contaminación radiactiva. También el desmantelamiento de las centrales nucleares, una vez que cumplieron su ciclo de vida útil, su depreciación nuclear, acaso, genera un alto grado de contaminación radiactiva. Se estima que todos estos residuos suman alrededor del $95 \%$ de la producción total de desechos radiactivos $^{2}$. También la contaminación radiactiva procede de aplicaciones no energéticas que derivan de los isótopos radiactivos como producto de las actividades industriales, investigativas y clínicas, esto es, procedentes de la medicina. Esta contaminación es menor y se estima en un grado "inferior al $10 \%$, sin que esto signifique que su gestión deba ser menos rigurosa" ("Residuos radiactivos", s.f.).

Si bien es cierto que la producción de energía nuclear conlleva grandes beneficios para la vida humana, también es cierto que la producción de esta basura nuclear causa daños irreversibles a la biosfera cuando no son debidamente tratados. Este es tan solo un problema más que enfrenta el medio ambiente como producto de las acciones humanas en el abastecimiento de sus necesidades.

\section{La heurística del temor de Hans Jonas}

\section{La amplitud del horizonte ético}

Según Jonas, "todo trato con el mundo extrahumano era éticamente neutral” (1995, p. 29). En realidad, la apelación a tiempos pasados no excluye que en la actualidad el imaginario de la neutralidad haya desaparecido. Es común escuchar en los salones de clase a los futuros científicos y tecnólogos opinar que, tanto la ciencia como la tecnología, son neutrales. El mundo extrahumano del que nos habla Jonas es, en efecto, neutral. Lo que no es neutral es la acción que sobre ese mundo hacen las personas.

2 Véase, Residuos radiactivos, s.f. 
La noción de extrahumano, que básicamente remite a la naturaleza, busca alcanzar un estatuto ético, mismo que le ha sido negado hasta ahora. "Lo que tenía relevancia ética era el trato directo del hombre con el hombre, incluido el trato consigo mismo; toda ética tradicional es antropocéntrica." (1995, p. 29). La ética tradicional, de acuerdo con el planteamiento de Jonas, "tenía que ver con el aquí y el ahora, con las situaciones que se presentan entre los hombres", se trataba de una ética centrada en el "entorno inmediato de la acción” en cuanto implicaba al mismo hombre (Jonas, 1995, p 30), de ahí la denominación de antropocéntrica. La naturaleza, como tal, no se vislumbra, aún, como objeto de consideración moral. Al ser una ética del aquí y el ahora de las acciones, el punto o eje de enjuiciamiento consistía en las repercusiones inmediatas de la comunidad humana. La ética hoy sigue esa orientación casuística de lo humano: "El universo moral se compone de los contemporáneos y su horizonte de futuro está limitado a la previsible duración de la vida" (Jonas, 1995, p. 30)

Por eso Jonas considera que "La técnica moderna ha introducido acciones de magnitud tan diferente, con objetos y consecuencias tan novedosos, que el marco de la ética anterior no puede ya abarcarlos" (1995, p. 32). Este marco ético del que nos habla Jonas es la ética tradicional, la ética de la virtud aristotélica, e incluso, la ética del imperativo categórico. La ética hoy debe redireccionarse y ampliar su horizonte valorativo.

De las acciones de gran magnitud, el ejemplo paradigmático que ofrece Jonas es el de "la tremenda vulnerabilidad de la naturaleza sometida a la intervención técnica del hombre” (1995, p. 32); misma que se hace evidente de acuerdo con los daños causados hoy. Las éticas anteriores no consideraban la naturaleza como un agente de diálogo, precisamente, porque no es considera como agente moral; o si se quiere, no es considerada como objeto de valoración moral. "La naturaleza, en cuanto responsabilidad humana, es sin duda un novum sobre el cual la teoría ética tiene que reflexionar" (Jonas, 1995, p. 33).

Considerar un nuevo agente participativo en el diálogo moral, demanda ampliar la visión kantiana del reino de los fines:

Esto implicaría que habría de buscarse no sólo el bien humano, sino también el bien de las cosas extrahumanas, esto es, implicaría ampliar el 
reconocimiento de «fines en sí mismos» más allá de la esfera humana e incorporar al concepto del bien humano el cuidado de ellos (Jonas, 1995, p. 35).

Desde luego, dentro de esta noción de extrahumanas cabe cualquier cosa, aunque Jonas hace un específico énfasis en la naturaleza.

\section{Heurística del temor}

Jonas nos invita a practicar una "ciencia de la predicción hipotética", llamada también, "futurología comparada" (Jonas, 1995, p. 64). Se trata de una proyección hipotética, de construcciones ético-filosóficas de lo que podría esperarse en el futuro de acuerdo con los cursos de acción hoy tomados. De ahí que Jonas diga que la proyección hipotética puede servir, bien para promover o bien para evitar cualquiera de estos cursos de acción.

Puntualmente, de acuerdo con la aplicación de esta futurología,

solamente la prevista desfiguración del hombre nos ayuda a forjarnos la idea del hombre que ha de ser preservada de tal desfiguración; y necesitamos que ese concepto se vea amenazado (...) para, ante el espanto que tal cosa nos produce, afianzar una imagen verdadera del hombre. Mientras el peligro es desconocido no se sabe qué es lo que hay que proteger y por qué; el saber acerca de ello procede, en contra de toda lógica y de todo método, de «aquello que hay que evitar» (Jonas, 1995, p.65).

¿En qué se basa Jonas para establecer esta futurología comparada? Básicamente Jonas establece la imaginación y el temor como componentes para idear la visualización de las repercusiones que puedan acarrear los cursos de acción hoy tomados. Muy acertadamente Jonas nos dice que "Solamente sabemos qué está en juego cuando sabemos que está en juego” (1995, p. 65). Pero, como no podemos saber qué está en juego en el futuro, entonces no podemos saber que el futuro, en este caso el de la humanidad, está en juego. Aquí es donde ingresa la imaginación (los experimentos mentales) para visualizar lo que está en juego y por qué está en juego.

Jonas nos invita a practicar "experimentos mentales" (1995, p. 68), como recurso de prever las repercusiones futuras de nuestros cursos de acción presentes. La tarea de los experimentos mentales hipotéticos no es la de 
presentar pruebas, sino ilustraciones. "Se trata, por tanto, de una casuística imaginaria cuya función no es, como en la casuística propia del derecho y la moral, poner a prueba principios ya conocidos, sino rastrear y descubrir lo todavía desconocido" (Jonas, 1995, p. 69).

La ciencia de la predicción hipotética es la capacidad de hacer experimentos mentales, imaginar el futuro de acuerdo con las acciones realizadas hoy por el ser humano. Sin embargo, una característica fundamental de estos experimentos mentales consiste en su visión catastrofista: es mejor representarse el malum antes que el bonum. A esto se le llamaría heurística del temor.

La heurística del temor de Jonas se basa en las proyecciones hipotéticas de un malum antes que de un bonum. Al respecto nos dice Jonas que "La mera presencia del mal nos impone su conocimiento, mientras que lo bueno puede pasar desapercibido y quedar ignorado sin que hayamos reflexionado sobre ello" (1995, p. 65).; y precisamente, como sabemos mucho antes "lo que no queremos que lo que queremos", es que "la filosofía moral tiene que consultar antes nuestros temores que a nuestros deseos, para averiguar qué es lo que realmente apreciamos" (1995, p. 66).

Para Jonas, "la guía del temor" es fundamental para la ética orientada al futuro. Dado que se trata de un horizonte lejano, donde "lo que ha de ser temido no es todavía experimentado y no encuentra quizás ninguna analogía en la experiencia pretérita y presente" (1995, p. 66). Aquí el papel del malum representado tiene que asumir el papel del malum experimentado; y esa representación no aparece por sí misma, sino que ha de ser procurada adrede" (1995, p. 66). Pero no se trata de un temor patológico, "sino de un temor de carácter espiritual", esto es, un temor, para parodiar a Savater, por amor propio, es decir, "una disposición a dejarse afectar por una felicidad o por una desgracia solamente representada de las generaciones venideras" (1995, p. 67).

Esta es la razón por la cual cree Jonas que solamente a través de la prevista desfiguración del hombre podríamos forjarnos la idea de hombre que ha de ser preservada de tal desfiguración. Como se aprecia, la heurística del temor de Jonas se basa en una cierta prudencia temerosa. Una visión catastrofista, pero no patológica, ayuda a recrear los experimentos mentales hipotéticos, cuya finalidad principal es advertir sobre los peligros de determinados cursos de acción tomados hoy. 


\section{Heurística de la razón instrumental}

¿Hasta qué punto la heurística del temor de Hans Jonas es racionalmente aceptable? Lo que está en juego es el futuro de la humanidad. La visión de Jonas sigue siendo, a su modo, antropocéntrica. En efecto, él mismo se plantea una interrogante al respecto: "¿Se trata simplemente de la prudencia que nos prohíbe matar la gallina de los huevos de oro o cortar la rama sobre la que uno está sentado?" (Jonas, 1995, p. 33). La respuesta que el mismo Jonas ofrece es de una preservación antropocéntrica, dado que lo que está en juego es el destino de la humanidad por su dependencia con la naturaleza: "ha de conservarse la orientación antropocéntrica de toda la ética clásica" (Jonas, 1995, p. 33). Desde luego, lo que está en juego es el ser humano, la naturaleza es el medio, no el fin. La ética continúa siendo antropocéntrica.

El futuro de la humanidad está en riesgo. Ante este inminente riesgo el recurso que ofrece Jonas es una proyección hipotética del temor, una heurística del temor enfocada desde el presente hacia el futuro.

Lo que cabe analizar es hasta qué punto el riesgo puede ser abordado desde esta heurística del temor. "Un análisis del riesgo no puede hacerse sin tomar en consideración aspectos teóricos, relacionados con la manera cómo se establecen las condiciones para la consideración de una situación o desarrollo como riesgo." (Vargas, 2004, p. 35). Si vemos la propuesta de Jonas, a la luz de esta observación de Celso Vargas, habremos de caer en cuenta que en Jonas existe todo un marco teórico de análisis en relación con los peligros potenciales de una civilización tecnológica al margen de una visión ética futurista y ampliada del ser humano y su forma de relacionarse con los demás seres extrahumanos.

El riesgo es la probabilidad de que se produzca un daño. Para considerar tal efecto pueden tomarse en cuenta la proyección de ciertas heurísticas. Celso Vargas menciona tres, las cuales, grosso modo, dicen que se tiene que tomar en cuenta las "predicciones que establece una teoría general, que sirven como base para una precisión posterior". Asimismo, los "modelos de simulación" pueden "determinar la evolución de un sistema como resultado de introducir determinadas alteraciones" (2004, p. 38). Finalmente el "uso de analogías", esto es, "incurrir a situaciones que presentan alguna similitud estructural o funcional puede ser valiosa para este propósito.” (2004, p. 38). 
Edgar Roy Ramírez señala que "Una forma de proceder en filosofía puede perfectamente ser el preguntarse cómo sería la realidad si tal o cual tesis filosófica fuera verdadera o se cumpliere" (2003, p. 88). Como añade el autor,

Se trata, en consecuencia, de analizar razones, la calidad de ellas y su coherencia interna (...). Se puede preferir por razones estéticas, de simplicidad, elegancia; o se puede rechazar una posición filosófica por embotamiento o por agotamiento teórico (2003, p.88).

A la luz de estas dos consideraciones, la heurística del temor cumple con algunos de estos presupuestos señalados por ambos autores costarricenses. Jonas invita a una lectura, cuyo corpus teórico descansa en una doctrina del ser de la naturaleza, y en consecuencia, un nuevo ethos para el ser humano, quien aún hoy no toma como objeto de valoración moral a la naturaleza y las generaciones futuras. Por otro lado, Jonas establece una serie de analogías predicativas en relación con los cursos de acción del ser humano del día. Si, como plantea Edgar Roy Ramírez, de lo que se trata es de "tomar en serio a los filósofos, de tomarlos como interlocutores dignos y de aceptar la pretensión de los filósofos de que algunas de sus aseveraciones son o pueden ser verdaderas" (2003, p. 88), lo que resta es una mayor divulgación de una cultura ética ambiental. Un sistema computacional podría representar la heurística del temor de Jonas en relación con los desastres ambientales debidos a malos cursos de acción en el presente. Esto sin embargo, ya se ha hecho independientemente de la lectura de Jonas. Lo importante es que el planteamiento filosófico como tal se ha adelantado, como siempre, a otras visiones de mundo, que por su popularidad suelen silenciar planteamientos más serios en orden a evitar desgracias humanas y ecológicas.

Los desechos radiactivos constituyen un problema ambiental significativo. No son los únicos problemas que le compete solucionar al ser humano, pero sin duda alguno, se trata de uno que involucra tanto los sectores urbanos como rurales y naturales.

La idea de una futurología comparada, de una heurística del temor aplicada a la razón instrumental, o bien a la racionalidad industrial, tendría como fin visualizar los daños que provocaría la alta contaminación de la industria sobre el medio ambiente. 
La racionalidad industrial es simplemente un ente que opera, como toda empresa o corporación, bajo un organigrama y en la que sus dueños o accionistas, solo requieren de los altos ejecutivos la máxima utilidad de la corporación en el mercado. Cualquier ejecutivo de alto rango que no logre incrementar la eficiencia, eficacia y la utilidad en la industria, simplemente será despedido. En el mundo empresarial no existe seguridad laboral.

Dada esta peculiar característica, un sector vulnerable lo constituye la sociedad, o bien el medio ambiente cuando no hay responsabilidad social de la empresa. La racionalidad industrial determina los cursos de acción económicos, y como no existe otro horizonte más que esta finalidad, la heurística del temor se hace necesaria ante el impacto negativo que podría acarrear una razón totalmente industrializada. Si tomáramos en serio a Jonas, tal y como plantea Edgar Roy Ramírez, la prevista desfiguración del medio ambiente, esto es, la prevista desfiguración del impacto ambiental que provoca la industria sin una orientación ecológica, nos ayuda a visualizar la idea del medio ambiente que necesitaríamos conservar.

Pese a que hoy sabemos qué es lo que está en juego, a saber, el medio ambiente y una parte considerable de su biodiversidad, y que además, los experimentos mentales que Jonas una vez ilustró con el elemental uso de la imaginación, y que hoy son recreaciones en fina realidad virtual, y aún así el ser humano hace caso omiso de las advertencias de un holocausto ecológico. Ni el mal, ni el bien son ya motivo de enderezar unos cursos de acción dañinos para la humanidad. Hoy parece que la presencia del mal no nos está imponiendo conocimiento alguno para direccionar el ethos destructivo que estamos cultivando a diario.

El ser humano está fagocitando de una forma irracional el único recurso que lo sustenta. La racionalidad industrial, en su afán de incrementar las utilidades que la sustentan, está devorando el recurso real que la mantiene en pie. A la interrogante de Jonas, de si es la prudencia que nos prohíbe matar la gallina de los huevos de oro o cortar la rama sobre la que estamos sentados, parece que ni siquiera la idea antropocéntrica es capaz de frenar el inminente holocausto ecológico que enfrentará la humanidad. La racionalidad del mercado es muy irracional como para ser prudente.

¿Qué pasaría si operáramos bajo el supuesto de consultar antes nuestros temores que a nuestros deseos? ¿Habría una mejor relación humana, ecológica, 
espiritual? ¿No caeríamos en una suerte de paranoia ecologizante? De acuerdo con la noción de riesgo tratada más arriba, consultar nuestros temores no constituye una forma paranoica de actuar. En esta suposición se está poniendo en juego la posibilidad real de un daño irreversible por anteponer en primer lugar los deseos de una lógica mercantil. El deseo se expresa como idea y el marketing hace posible la materialización de esta idea bajo los imperativos del deseo irracional, para averiguar qué es lo que realmente apreciamos.

Tómese en consideración el depósito de material radiactivo en los llamados basureros nucleares. La industria necesita la explotación del uranio porque existe un mercado meta para los productos y servicios que se derivan de él. El problema, como se vio, consiste en los desechos o residuos que quedan una vez utilizados en las plantas nucleares. De acuerdo con la heurística del temor de Jonas, y con la ciencia de la predicción hipotética, analicemos un caso hipotético.

Los desechos radiactivos son almacenados, en el mejor de los casos, a cientos de metros bajo tierra, en un lugar capaz de contener tales desechos el tiempo suficiente para impedir que la radiación contamine el ambiente. Sin embargo, las filtraciones radiactivas no solamente son una posibilidad lógica, sino también técnica. Si por alguna razón estos muros de contención no lograsen impedir la irradiación, o por otro lado, se presentaran filtraciones de agua que pudieran arrastrar isótopos radiactivos fuera del vertedero o fosa nuclear hacia las fuentes hídricas, se desencadenaría una contaminación radiactiva inminente. De la misma forma, el agua de lluvia o el agua subterránea podrían entrar en contacto con los materiales radiactivos provocando fuga radiactiva. La posibilidad de que el agua entre en contacto con los desechos radiactivos y que logren disolverse algunos de los radionúclidos y luego viajar a través de la venas hídricas hacia la superficie terrestre, podría poner en peligro la vida de muchas criaturas biológicas, incluyendo al ser humano.

Ahora habría que analizar con expertos en la materia, si el experimento mental, esto es, si la heurística del temor aquí usada, tiene fundamentos o no. Lo cierto es que la sola posibilidad de una catástrofe humana hace que revaloremos lo que hoy estamos realizando como ecológicamente correcto. La heurística del temor funciona siempre que exista la humildad y el compromiso de las grandes corporaciones de escuchar la voz de algún agente interesado en la preservación de nuestro medio ambiente. 


\section{Referencias}

Badillo Álvarez, V. y Pérez, J. (2004). Desechos nucleares: ¿de dónde vienen y hacia dónde van? Ciencia, abril-junio. Versión digital tomada de: http://agalano.com/Cursos/ MetExpI/desechos_nucleares.pdf

Coronado, G. (2002). La ciencia, la técnica y la tecnología. En Álvaro Zamora y Guillermo Coronado (comps). Perspectivas en ciencia, tecnología y ética. Cartago: Editorial Tecnológica de Costa Rica.

Jonas, H. (1995). El principio de responsabilidad (Trad. Javier Fernández Retenaga). Barcelona: Herder.

Mora, G. (2003). Racionalidad y tipos de racionalidad. Revista de Filosofía de la Universidad de Costa Rica. XLI, (103), 93-100.

Ramírez, E. R. (2003). ¿Cómo evaluar posiciones filosóficas? Revista de Filosofía de la Universidad de Costa Rica. XLI, (103), 87-93.

Residuos radiactivos (s. f.). Consultado en: http:/www.tecnun.es/asignaturas/Ecologia/ Hipertexto/13Residu/150ResRadi.htm

Residuos nucleares en almacenes geológicos profundos (s. f.). Consultado en www.econonoticias.com

Residuos nucleares: ¿qué hacer con ellos? (s. f.) Consultado en: http://revista.consumer.es/ web/es/20040301/medioambiente/68171.php

Rodríguez, F. (2002). La naturaleza caída. San José, C. R.: Ediciones Perro Azul.

Vargas, C. (2004). Análisis epistemológico del riesgo. Revista de Filosofía de la Universidad de Costa Rica. XLII, (106-107), 35-42 